\title{
Evaluating the width to length ratio of maxillary anterior teeth in Class I canine relation- An observational study
}

\author{
Dweepika Garg* and Santosh K Goje \\ Department of Orthodontics and Dentofacial Orthopaedics, K.M Shah Dental College and Hospital, Sumandeep Vidyapeeth, Gujarat, India
}

\begin{abstract}
Introduction: Maxillary lateral incisor is the most common tooth that is prone to deformity in context with size, shape and texture. The major goal of orthodontic treatment for the great majority of patients in recent years is dental and facial aesthetics. To achieve goals in orthodontics, it is necessary to have canines in a good Class I relationship. The mean width/length ratio of the maxillary 3 anterior tooth groups is 0.81 . Understanding the relationship between the width and length of the clinical crown of normal teeth might be useful in helping establish a more precise endpoint when managing a case of altered passive eruption. This would help us in achieving functional stability along with esthetic harmony.
\end{abstract}

Aims and objectives: To determine width to length ratio of maxillary anterior teeth and correlate it with class I canine relation.

Materials and methods: Alginate impressions of 18 to 25 years old individuals who fulfill the inclusion criteria will be taken to measure the width and length of maxillary six anterior teeth.

Results: The values obtained are not statistically significant; indicating that there is no correlation between class I canine relation and the universally accepted 0.81 width to length ratio of the three maxillary anterior teeth.

Conclusion: Class I canine relation does not necessarily guarantee establishment of 0.81 width to length ratio. So even if the functional stability is achieved at the end of the treatment by achieving a class I canine relation, the anterior teeth might still not follow 0.81 width to length ratio, in spite of being in proper occlusion.

\section{Introduction}

For many years, the establishment of health and function was the primary objective of dental professionals. However, the goal of modern dentistry includes not only health and function, but also a final aesthetic result corresponding to the shape, size, colour, texture, and harmony of natural healthy teeth and their surrounding tissues. Additional factors that might influence the ideal smile are ethnicity, personality, size, and position of teeth and clinical crowns, or simply the perception of what is "ideal" by a specific group or population [1].

The relative dimensions of teeth seem to be among the most objective dental criteria within the aesthetic checklist because they can be easily and physically controlled. The definition of ideal tooth dimensions, however, remains a difficult task due to individual variations and proximal/incisal tooth wear. To provide "magic numbers" for the clinician, mathematic theorems such as the "golden proportion" and the "golden percentage" have been proposed, considering classic elements of art and architecture. These rules were applied to the apparent size, as viewed directly from the anterior [1] (Figure 1).

Maxillary lateral incisor is the most common tooth that is prone to deformity in context with size, shape and texture. Since it is often subjected to variation, studies have been conducted to suggest ideal width of maxillary lateral incisor. From the lateral incisor perspective, various widths were suggested. The width of the lateral incisor should be $62 \%$ of the width of the maxillary central incisor (Figure 2). Width of each tooth to the total width of the anterior teeth (canine to canine) should be as follows: Canine 10\%, lateral incisor $15 \%$ and central incisor 25\% [2].
According to Jackson's Triad, goal of orthodontic treatment is to achieve: Functional Efficacy, Aesthetic harmony and structural balance. The major goal of orthodontic treatment for the great majority of patients in recent years is dental and facial aesthetics. This goal is not "just cosmetic". It reflects the patients' desire to improve their social acceptability and eliminate discrimination based on appearance, which greatly affect their quality of life [3].

To achieve goals in orthodontics, it is necessary to have canines in a good Class I relationship i.e. the mesial incline of the maxillary canine occludes with the distal incline of mandibular canine.

There is a variation on dental dimensions that can be considered as normal or desirable, especially when considering that there are individuals with different facial patterns. The dimensions of anterior teeth can be defined within a limited range. The height of the central incisor varies from 10.4 to $11.2 \mathrm{~mm}$ while its width varies from 8.73 to $9.3 \mathrm{~mm}$, and, usually, these references are used in prosthetic reconstructions, when no other parameters are available. The most important is the correlation of these dimensions, i.e., the dental proportions. Two proportions must be considered: the relation between

${ }^{\star}$ Correspondence to: Dweepika Garg, Department of Orthodontics and Dentofacial Orthopaedics, K.M Shah Dental College and Hospital, Sumandeep Vidyapeeth, Gujarat, India, E-mail: dweepika_dg1@yahoo.co.in

Key words: width to length ratio, class I canine relation, vernier calipers

Received: September 21, 2018; Accepted: October 25, 2018; Published: October 30,2018 

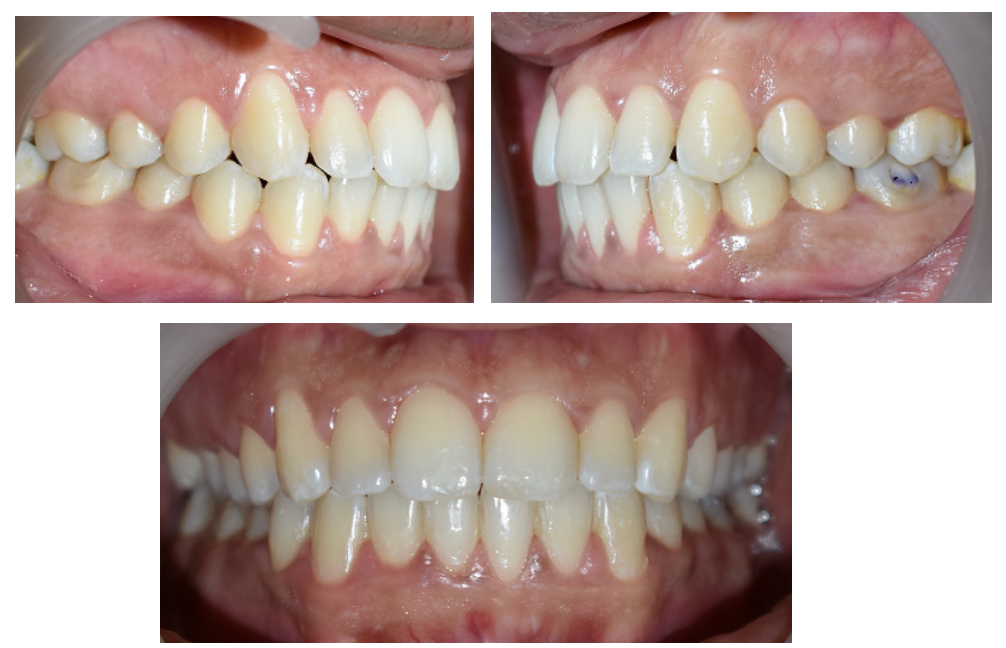

Figure 1. Intraoral view of participant selected for the study

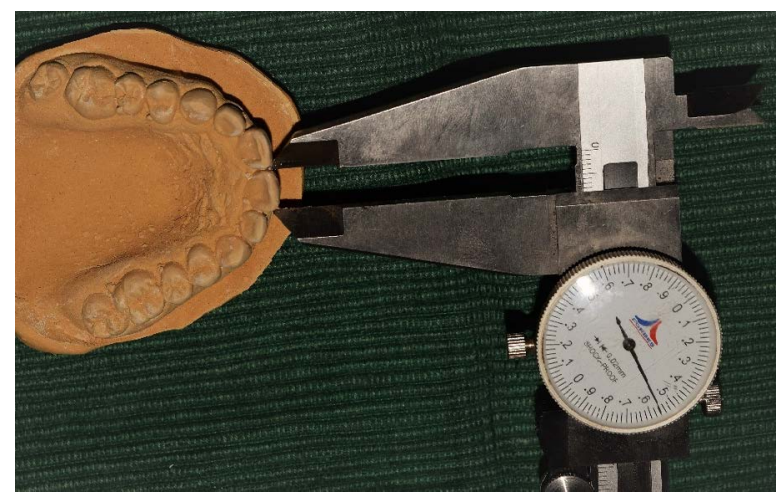

Figure 2. Vernier calipers to measure the width of central incisor

height and width of each tooth, and the relation of height and width among the teeth [4].

Sterrett et al. [5] carried out an investigation to analyse the clinical crown of the three tooth groups of the maxillary anterior sextant of the permanent dentition of normal subjects with respect to width, length and the width/length ratios and found correlation between tooth dimensions or tooth group ratios and subject height. They concluded that the mean width/ length ratio of the maxillary 3 anterior tooth groups is 0.81 .

Alsulaimani and Batwa in 2013 [2] conducted a study to determine whether alteration of the maxillary central and lateral incisors' length and width, respectively, would affect perceived smile aesthetics. Photographic manipulation was undertaken to produce two sets of photographs, each set of four photographs showing the altered width of the lateral incisor and length of the central length. The eight produced photographs were assessed by laypeople, dentists and orthodontists. They concluded that all participants recognized that the central incisors' length changes. For lateral incisors, laypeople were more tolerant than dentists and orthodontists. This suggests that changing incisors' proportions affects the relative smile attractiveness (Figure 3).

Khan and Hussain in 2015 [1] aimed to determine clinical crowns length, width and width/length ratios of the maxillary anterior teeth. They concluded that the tooth dimension showed less variation (low standard deviation value) for crown length, width, and width/length ratio; so, it will help the clinician in restorative procedures.
After searching the literature databases like PubMed, Medline, Ebsco, Scopus, LILACS, till May 2018, with no restriction on language; no study was found which checked the validity of 0.81 width/length ratio of maxillary anterior teeth when canine is in class I relationship. The mean width/length ratio of the maxillary 3 anterior tooth groups is 0.81 . Understanding the relationship between the width and length of the clinical crown of normal teeth might be useful in helping establish a more precise endpoint when managing a case of altered passive eruption. This would help us in achieving functional stability along with esthetic harmony. Therefore, this study was taken up.

The aim of the study was to determine width to length ratio of maxillary anterior teeth and correlate it with Class I canine relation.

\section{Materials}

The study was conducted in Department of Orthodontics, K. M. Shah Dental College and Hospital, Piparia, Vadodara. 18 to 25 years old participants who fulfill the inclusion criteria were included from the reception OPD of K. M. Shah Dental College and Hospital, Piparia, Vadodara.

Based upon the study done by Khan [1], the sample size is calculated. The minimum sample required for the study is 25 . The sample size of the study is 100 .

The inclusion criteria were- all of the 6 maxillary anterior teeth should be present, and the anatomic elements should be well defined, Matching midlines, Class I canine relationship, Participants should be in the age range of 18 to 25 years. 
Participants were excluded if there were any signs of gingival alteration, hyperplasia, inflammation, those with restorations, traumatisms, attrition, occlusal adjustment, dental malformation, spacing in upper anterior region, restoration in any of the anterior teeth, history of previous orthodontic treatment or any esthetic correction.

\section{Methods}

Participants fulfilling the inclusion criteria were selected from the OPD of KMSDCH. After taking written informed consent form, alginate impressions were made of upper arch and lower arch. Then study casts were prepared from plaster.

An extra-fine end digital caliper was used to obtain the measurements, with a precision of $0.01 \mathrm{~mm}$. The measurements were recorded in millimeters by the principal investigator. The maximum mesiodistal width (perpendicular to the longitudinal axis of the tooth) and the clinical crown length (between the most apical point of the gingival margin and the most incisal point of the anatomic crown) of the maxillary central incisors, lateral incisors, and canines were recorded for each tooth. The data was arranged in a table and the data obtained was subjected for statistical analysis.
One-way Anova (Table 1) shows that the mean W/L ratio of central incisor is 0.863 ; the mean $\mathrm{W} / \mathrm{L}$ ratio of lateral incisor is 0.881 whereas the mean $\mathrm{W} / \mathrm{L}$ ratio of canine is 0.870 . The within groups and between groups Anova tests shows that the $p$ value is 0.633 ; indicating the data obtained is not statistically significant. Multiple comparisons are done using the Post Hoc tests (Table 2).

When the W/L ratio of central incisor, lateral incisor and canine are compared with each other, the $p$ value obtained is more than $0.05 \%$. This shows that there are no significant differences in the ratios of the three anterior teeth (Figure 4).

\section{Discussion}

This study was carried out with the aim of determining the width to length ratio of maxillary anterior teeth and correlate it with Class I canine relation. In recent years, aesthetic dentistry has become a major focus for the public. Final aesthetic result corresponding to the shape, size, colour, texture, and harmony of natural healthy teeth and their surrounding tissues. Orthodontics plays a pivotal role in enhancing facial aesthetics of an individual.

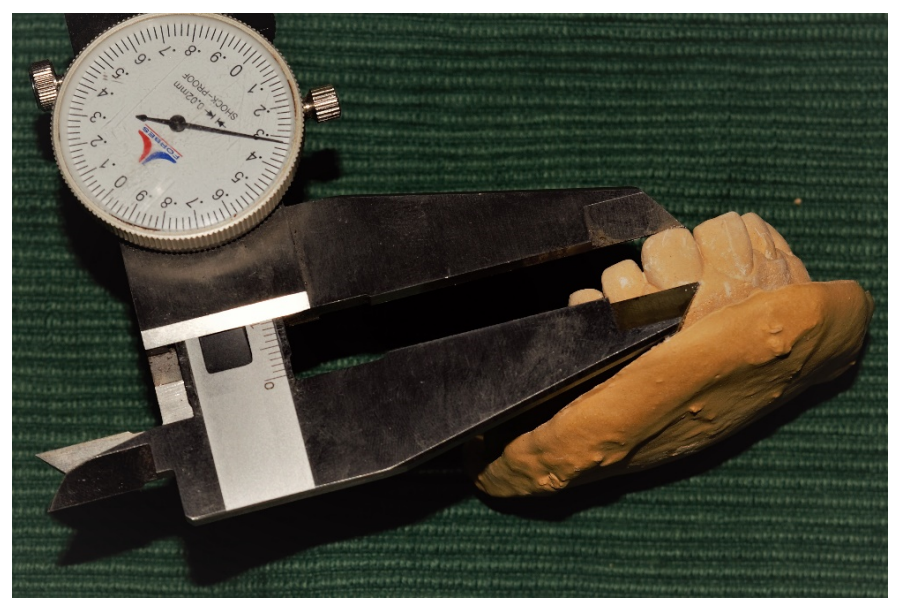

Figure 3. Vernier calipers to measure the length of central incisor

Table 1: Oneway ANOVA

\begin{tabular}{|c|c|c|c|c|}
\hline DATA & Sum of Squares & df & Mean Square & ANOVA P VALUE \\
\hline Between Groups & 0.016 & 2 & 0.008 & 0.458 \\
\hline Within Groups & 5.060 & 297 & 0.017 & \\
\hline Total & 5.076 & 299 & & \\
\hline
\end{tabular}

Table 2: PostHoc tests

\begin{tabular}{|c|c|c|c|c|c|c|}
\hline \multicolumn{7}{|c|}{ Multiple Comparisons } \\
\hline \multicolumn{7}{|c|}{$\begin{array}{c}\text { Dependent Variable: DATA } \\
\text { Tukey HSD }\end{array}$} \\
\hline \multirow{2}{*}{\multicolumn{2}{|c|}{ (I) GROUP }} & \multirow{2}{*}{ Mean Difference (I-J) } & \multirow{2}{*}{ Std. Error } & \multirow{2}{*}{ P VALUE } & \multicolumn{2}{|c|}{$95 \%$ Confidence Interval } \\
\hline & & & & & Lower Bound & Upper Bound \\
\hline \multirow{2}{*}{$\begin{array}{l}\mathrm{W} / \mathrm{L} \text { ratio of central } \\
\text { incisor }\end{array}$} & $\begin{array}{l}\mathrm{W} / \mathrm{L} \text { ratio of lateral } \\
\text { incisor }\end{array}$ & -.018 & 0.018 & 0.607 & -0.061 & 0.026 \\
\hline & $\mathrm{W} / \mathrm{L}$ ratio of canine & -.007 & 0.018 & 0.915 & -0.051 & 0.036 \\
\hline \multirow{2}{*}{$\begin{array}{l}\mathrm{W} / \mathrm{L} \text { ratio of lateral } \\
\text { incisor }\end{array}$} & $\begin{array}{l}\mathrm{W} / \mathrm{L} \text { ratio of central } \\
\text { incisor }\end{array}$ & .018 & 0.018 & 0.607 & -0.026 & 0.061 \\
\hline & $\mathrm{W} / \mathrm{L}$ ratio of canine & .010 & 0.018 & 0.845 & -.033 & 0.054 \\
\hline \multirow{2}{*}{$\mathrm{W} / \mathrm{L}$ ratio of canine } & $\begin{array}{l}\mathrm{W} / \mathrm{L} \text { ratio of central } \\
\text { incisor }\end{array}$ & 0.007 & 0.018 & 0.915 & -0.036 & 0.051 \\
\hline & $\begin{array}{l}\mathrm{W} / \mathrm{L} \text { ratio of lateral } \\
\text { incisor }\end{array}$ & -0.010 & 0.018 & 0.845 & -0.054 & 0.033 \\
\hline
\end{tabular}




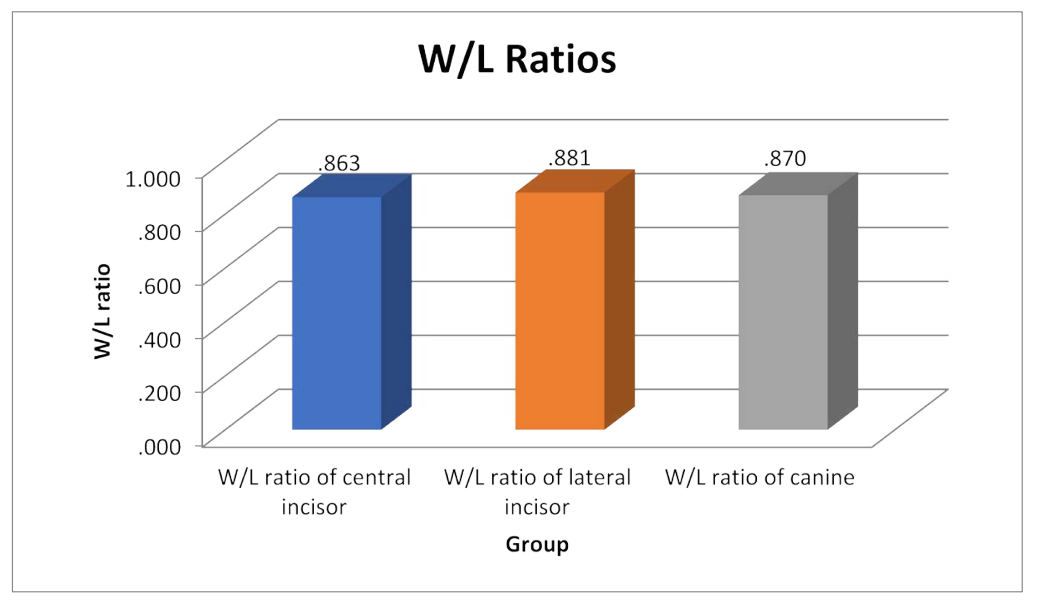

Figure 4. W/L ratio of central incisor, lateral incisor and canine

Sterrett et al. [5] in 1998 had carried out a study to know the mean width to length ratio of anterior teeth. They had concluded that within male and female Caucasians, the mean width/ length ratio of the maxillary 3 anterior tooth groups is 0.81 . This 0.81 width to length ratio is highly accepted and widely used.

Till now no study was found which checked the validity of 0.81 width/length ratio of maxillary anterior teeth when canine is in class I relationship in Indian population. Therefore, this study was taken up.

Study cast of 18 to 25 years old individuals with Class I canine relation were obtained. They fulfilled all the inclusion criteria set for the study. The width to length ratio was calculated for the 3 anterior teeth.

All the values obtained were subjected to statistical analysis. It was found that class I canine relation with matching midlines, does not fulfill 0.81 width to length ratio. So, even if the maxillary and mandibular canines are in class I relation, it does not guarantee ideal proportion of anterior teeth.

Muhammad et al. [1] in their study found out that the mean crown width/length ratio was 0.91 for central incisor, 0.72 for lateral incisor and 0.71 canines. However, in our study, the mean W/L ratio of central incisor is $0.863,0.881$ for lateral incisor and 0.870 for canine.

Anterior teeth are essential for esthetics in Orthodontics, Prosthodontics and other restorative patients. Knowing the mean values of width to length will help the clinician to adequately restore these teeth. Fahad et al. [2] had concluded that changing the maxillary central incisor length, the maxillary lateral incisor width did influence the smiles esthetics. As the central incisor's length is reduced, the smile arc appears flatter, and the flatter the smile arc, the least esthetic it becomes. Thus, the proportions of teeth play a very important role in esthetics.

The maxillary lateral incisor is the most common tooth that shows variation in its size and shape, where it may be called diminutive, peg shaped lateral or, in some circumstances, it may fail to develop altogether. Understanding the relationship between the width and length of the clinical crown of normal teeth is useful in establishing a more precise end point when managing a case of altered passive eruption. If the canine is displaced bucally or palatally and the lateral incisor is peg shaped, by knowing the width to length ratio, build-up of peg lateral can be done. This helps in achieving functional stability along with esthetic harmony.
So even if the functional stability is achieved at the end of the treatment by achieving a class I canine relation, it is not necessary that anterior teeth might still follow the 0.81 width to length ratio, in spite of being in proper occlusion. Thus, in study, the width to length ratio of anterior teeth were determined and were correlated with class I canine relation. It is concluded that there is no correlation between the two. Achieving Class I canine relation will not guarantee establishment of 0.81 width to length ratio.

The relative dimensions of teeth can be easily and physically controlled. The major goal of orthodontic treatment for the great majority of patients is dental and facial aesthetics. Thus, the two proportions must be considered: the relation between height and width of each tooth, and the relation of height and width among the teeth.

\section{Conclusion}

It can be concluded that Class I canine relation does not necessarily guarantee establishment of 0.81 width to length ratio. Class I canine relation is independent of the width to length ratio of anterior teeth.

\section{References}

1. Khan M, Khan MA, Hussain U (2015) Clinical crown length, width and the width/ length ratio in the maxillary anterior region in a sample of mardan population. Pak Oral \& Dent J 4: 738-741.

2. Alsulaimani FF, Batwa W (2013) Incisors' proportions in smile esthetics. J Orthodont Sci 2: 109-112. [Crossref]

3. Bansal A, Jain A, Patel S, Naik A, Deshmukh C, et al. (2015) Mini and Micro Esthetics in Orthodontics: Review on Clinical Considerations in Orthodontic Diagnosis. Arch of Dent and Med Res 1: 32-39.

4. Brandao RCB, Brandão LBC (2013) Finishing procedures in Orthodontics: dental dimensions and proportions (microesthetics). Dental Press J Orthod 18: 147-174. [Crossref]

5. Sterrett JD, Oliver T, Robinson F, Fortson W, Knaak B, et al. (1999) Width/ length ratios of normal clinical crowns of the maxillary anterior dentition in man. $J$ Clin Periodontol 26: 153-157. [Crossref]

Copyright: (C2018 Garg D. This is an open-access article distributed under the terms of the Creative Commons Attribution License, which permits unrestricted use, distribution, and reproduction in any medium, provided the original author and source are credited. 